\title{
Uniqueness of electrical currents in a network of finite total resistance*
}

\author{
Agelos Georgakopoulos ${ }^{\dagger}$ \\ Technische Universität Graz \\ Steyrergasse 30, 8010 \\ Graz, Austria \\ Mathematics Subject Classification: 05C21, 05C80
}

\begin{abstract}
We show that if the sum of the resistances of an electrical network $N$ is finite, then there is a unique electrical current in $N$ provided we do not allow, in a sense made precise in the paper, any flow to escape to infinity.
\end{abstract}

\section{Introduction}

Electrical networks are physical objects but also useful tools in mathematics. For example, they are closely related to random walks [24, 33, they find applications in the study of Riemannian manifolds $22,23,26$, and they are related to various problems in combinatorics 4. An electrical network $N$ has an underlying graph $G$ and a function $r: E(G) \rightarrow \mathbb{R}^{+}$assigning resistances to the edges of $G$. If $G$ is finite, then the electrical current in $N$-between two fixed vertices $p, q$ and with fixed intensity $I$ - is the unique flow satisfying Kirchhoff's cycle law, which demands that the potential differences sum to zero along every cycle of the graph. Recall that a flow in a graph by definition satisfies Kirchhoff's node law, which demands that current is preserved at every vertex other than $p$ and $q$. See Section 2 for more precise statements of these laws. If $G$ is infinite then several such flows may exist, and one of the standard problems in the study of infinite electrical networks is to specify under what conditions such a flow is unique, see e.g. [29, 31].

Our main result is that if the sum of all resistances in a network $N$ is finite, then there is a unique electrical current in $N$, provided we do no allow any flow to escape to infinity; more precisely, we require that for every finite edge-cut $F$ of $G$ that does not separate the source $p$ from the sink $q$ the net flow through $F$ is zero. We call a flow satisfying this condition non-elusive. To see the necessity of this requirement consider the network of Figure1. This network admits several

\footnotetext{
*Published in Journal of the L.M.S. Vol. 82 Nr. 1.

†Supported by a grant of the German-Israeli Foundation and by FWF grant P-19115-N18. This work was conceived and partly written when the author was a postdoc at the University of Hamburg.
} 


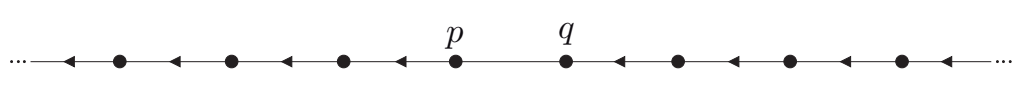

Figure 1: A flow escaping to infinity

flows, all of which satisfy Kirchhoff's cycle law as there are no cycles: one of these flows runs only along the edge $p q$; another can be obtained by sending a flow from $p$ all the way to the left end, and collecting the same amount of flow from the right end into $q$ as depicted in Figure 1. We consider the latter flow to be rather pathological, since it is permitted even if we disconnect the graph by removing the edge $p q$. And indeed, it is not a non-elusive flow. The interested reader will experiment with more complicated networks, and convince himself that requiring a flow to be non-elusive is a natural way of preventing a flow from using infinity in an abusive manner. It is worth remarking that in an 1-ended graph every flow is non-elusive. Non-elusive flows allowed a generalisation of the well-known Max-Flow Min-Cut theorem to infinite networks [1] (they were called finite-cut-respecting flows in the latter paper).

We can now state our main result:

finr Theorem 1.1. Let $N=(G, r, p, q, I)$ be a locally finite network with $\sum_{e \in E} r(e)<$ $\infty$. Then there is a unique non-elusive $p-q$ flow with intensity $I$ and finite energy in $N$ that satisfies Kirchhoff's cycle law.

The energy of a flow $f$ is defined by $W(f):=\sum_{e \in E(G)} f^{2}(e) r(e)$. The requirement that the energy be finite is necessary in the above statement (see Section (5), and very common in the literature.

The essential part of Theorem 1.1 is the uniqueness rather than the existence of the desired flow. Let us briefly consider the case when $G$ is finite. To prove uniqueness in that case, suppose there are two flows with the required properties, and consider their difference $z$. Then $z$ is a circulation in $G$, and so there must be some cycle along which $z$ is always positive in the same direction. But such a cycle yields a contradiction to the fact that $z$ must satisfy Kirchhoff's cycle law being the difference of two flows that do. Now back to the case when $G$ is infinite, the above argument breaks down as an infinite circulation need not traverse any finite cycle in the same direction. However, it is possible to prove that if a circulation is non-elusive, then it must traverse some (finite or infinite), topological circle; that is, a homeomorphic image of the real unit circle $S^{1}$ in the end-compactification $|G|$ of $G$. Such circles, introduced by Diestel and Kühn [13, have been the object of intense study recently [10, Section 8.5], and some of the acquired machinery is used here; see Section 2.1 for more about circles. As an intermediate result we obtain that these circles must also satisfy Kirchhoff's cycle law if all finite cycles do.

Our main result also relies on some recent results from [16] about topologies on graphs induced by an assignment of lengths to the edges; see Section 2.3 for details.

The proof of Theorem 1.1 spans Sections 3 to 5 In Section 6 we discuss the complementary case of finite total conductance. In Section 7 we extend to nonlocally-finite graphs. Finally, in Section 8 we discuss the relation of our results to stochastic processes and offer a conjecture related to the Dirichlet problem. 


\section{Definitions and basic facts}

We will use the terminology of Diestel [10] for graph theoretical terms and the terminology of 2] for topological ones.

A network is a tuple $N=(G, r, p, q, I)$, where $G$ is an (undirected) (multi-) graph, $r$ is a mapping assigning a resistance $r(e) \in \mathbb{R}^{+}$to each edge $e$ of $G$, $p, q \in V(G)$, and $I \in \mathbb{R}$ is a constant. A flow in $N$ is a real-valued $p-q$ flow in $G$ with intensity $I$ (intuitively $p$ and $q$ are connected to a battery generating a constant current of intensity $I$ ). A flow $f$ satisfies, by definition, Kirchhoff's node law at every vertex except $p$ and $q$, and the net flow leaving $p$ is the intensity of $f$. More formally, we have

Kirchhoff's node law: For every vertex $x \in V(G)$ there holds

$$
\sum_{x y \in E(G)} f(x, y)= \begin{cases}0 & \text { if } x \neq p, q \\ I & \text { if } x=p, \\ -I & \text { if } x=q .\end{cases}
$$

(Recall that $f$ is a function from $V^{2}$ to $\mathbb{R}$ satisfying $f(x, y)=-f(y, x)$ for every $x y \in E(G)$.) A circulation is a flow of intensity 0 .

A flow $f$ in $N$ is called non-elusive if for every finite cut $\left(X, X^{\prime}\right)$ such that both $p, q$ lie in $X$ (or both lie in $X^{\prime}$ ) there holds $f\left(X, X^{\prime}\right)=0$, where $f\left(X, X^{\prime}\right)=$ $\sum_{x y \in E, x \in X, y \in X^{\prime}} f(x, y)$. It follows that if $p \in X$ and $q \in X^{\prime}$ then $f\left(X, X^{\prime}\right)=$ $f(\{p\}, V(G)-p)=-f(\{q\}, V(G)-q)=I$. Note that if $G$ is finite or 1-ended (see Section 2.1 for the definition of an end) then every flow is non-elusive.

If $G$ is finite, then one defines the electrical current $i$ to be the flow in $N$ satisfying Kirchhoff's cycle law, and it is well-known - and not hard to provethat this flow always exists and that it is unique.

A directed cycle is a cycle together with a choice of one of its two possible orientations. If $C$ is a directed cycle then we let $\vec{E}(C)$ denote the set of ordered pairs $(x, y)$ such that $x y$ is an edge of $C$ traversed from $x$ to $y$ in the chosen orientation of $C$.

Kirchhoff's cycle law: For every directed cycle $C$ in $G$ there holds

$$
\sum_{\vec{e} \in \vec{E}(C)} v(\vec{e})=0
$$

where $v(\vec{e}):=i(\vec{e}) r(\vec{e})$ is the voltage drop or potential difference induced by $i$ along $e$ (in physics the equation $v(\vec{e})=i(\vec{e}) r(\vec{e})$ is known as Ohm's law).

An important concept for both finite and infinite electrical networks is that of energy: the energy of the flow $f$ is defined by $W(f):=\sum_{x y \in E(G)} f^{2}(x, y) r(e)$. $(W(f)$ is usually called "energy" in mathematics, but in physics it is called "power".)

The following theorem is well known, and indicates the importance of the concept of energy for electrical networks. See Section 4 for a proof.

exist Theorem 2.1. Let $N$ be a finite network and let $W=W(I, p, q)$ be the infimum of $W(f)$ over all flows $f$ in $N$. Then, there is a unique flow $i$ in $N$ satisfying $W(i)=W$. This flow satisfies Kirchhoff's cycle law. 


\subsection{Ends, the Freudenthal compactification and wild cir- cles in graphs}

Let $G$ be a graph, fixed throughout this section.

A 1-way infinite path is called a ray, a 2-way infinite path is a double ray. A tail of a ray $R$ is an infinite (co-final) subpath of $R$. Two rays $R, L$ in $G$ are equivalent if no finite set of edges separates them. The corresponding equivalence classes of rays are the ends of $G$. We denote the set of ends of $G$ by $\Omega=\Omega(G)$.

We now endow the space consisting of $G$, considered as a 1-complex, and its ends with the topology $|G|$. Firstly, every edge $e \in E(G)$ inherits the open sets corresponding to open sets of $[0,1]$. Moreover, for every finite edge-set $S \subset E(G)$, we declare all sets of the form

$$
C(S, \omega) \cup \Omega(S, \omega) \cup E^{\prime}(S, \omega)
$$

to be open; here, $C(S, \omega)$ is any component of $G-S$ and $\Omega(S, \omega)$ denotes the set of all ends of $G$ having a ray in $C(S, \omega)$ and $E^{\prime}(S, \omega)$ is any union of halfedges $(z, y]$, one for every edge $e=x y$ in $S$ with $y$ lying in $C(S, \omega)$. Let $\|G\|$ denote the topological space of $G \cup \Omega$ endowed with the topology generated by the above open sets. Moreover, let $|G|$ denote the space obtained from $\|G\|$ by identifying any two points that have the same open neighbourhoods. (Our notation is slightly non-standard: our "ends" are usually called edge-ends in the literature, and the symbol $|G|$ often denotes a different space if $G$ is nonlocally-finite. However, for a locally finite $G$ these differences disappear and our notation agrees with the mainstream.) If a point $x$ of $|G|$ resulted from the identification of a vertex with some other points (possibly also vertices), then, with slight abuse of notation, we still call $x$ a vertex. It is easy to see that two vertices $v, w$ of $G$ are identified in $|G|$ if and only if there are infinitely many edge-disjoint $v-w$ paths.

It it well-known (see [12]) that $|G|$ coincides with the Freudenthal compactification [15] of the 1-complex $G$ if $G$ is locally finite.

The study of $|G|$, in particular of topological circles therein, has been a very active field recently. It has been demonstrated by the work of several authors $([5,6,7,8,13,14,18,17,19,30)$ that many well known results about paths and cycles in finite graphs can be generalised to locally finite ones if the classical concepts of path and cycle are interpreted topologically, i.e. replaced by the concepts of a (topological) arc and circle in $|G|$; see Section 2.2 for some definitions and [10, Section 8.5] for an exposition of this field. An example of such a topological circle is formed by a double ray both rays of which converge to the same end together with that end. There can however be much more exciting circles in $|G|$ : in Figure 2, the $\aleph_{0}$ many thick double rays together with the continuum many ends of the graph combine to form a single topological circle $W$, the so-called wild circle. The double rays are arranged within $W$ like the rational numbers within the reals: between any two there is a third one; see 13 for a more precise description of $W$.

For a circle $C$ we let $E(C)$ denote the set of edges it traverses. We define directed circles and the notation $\vec{E}(C)$ similarly to the case when $C$ is a finite cycle. 


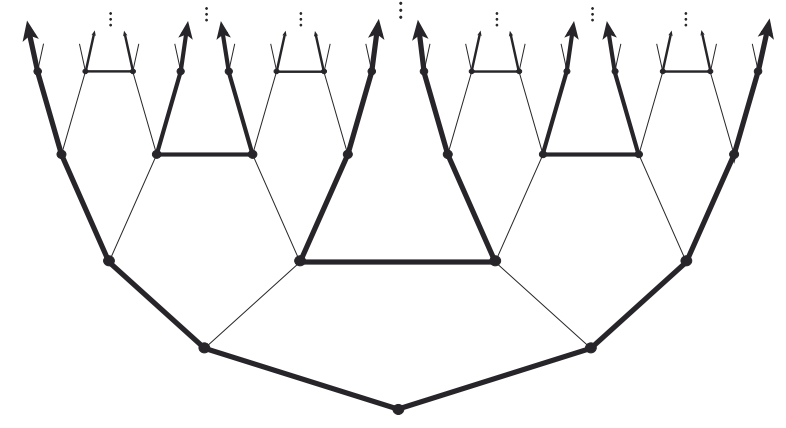

Figure 2: The 'wild' circle of Diestel and Kühn, formed by infinitely many (thick) double rays and continuum many ends.

\subsection{Topological paths, circles, etc.}

A circle in a topological space $X$ is a homeomorphic copy of the unit circle $S^{1}$ of $\mathbb{R}^{2}$ in $X$. An arc $R$ in $X$ is a homeomorphic image of the real interval $[0,1]$ in $X$. Its endpoints are the images of 0 and 1 under any homeomorphism from $[0,1]$ to $R$. If $x, y \in R$ then $x R y$ denotes the subarc of $R$ with endpoints $x, y$. A topological path in $X$ is a continuous map from a closed real interval to $X$.

Let $\sigma:[a, b] \rightarrow X$ be a topological path in a metric space $(X, d)$. For a finite sequence $S=s_{1}, s_{2}, \ldots, s_{k}$ of points in $[a, b]$, let $\ell(S):=\sum_{1<i<k} d\left(\sigma\left(s_{i}\right), \sigma\left(s_{i+1}\right)\right)$, and define the length of $\sigma$ to be $l(\sigma):=\sup _{S} \ell(S)$, where the supremum ranges over all finite sequences $S=s_{1}, s_{2}, \ldots, s_{k}$ with $a=s_{1}<s_{2}<\ldots<s_{k}=b$. If $C$ is an arc or a circle in $(X, d)$, then we define its length $l(C)$ to be the length of a surjective topological path $\sigma:[0,1] \rightarrow C$ that is injective on $(0,1)$; it is easy to see that $l(C)$ does not depend on the choice of $\sigma$.

\section{$2.3 \quad \ell-T O P$}

Fix a graph $G$ and a function $\ell: E(G) \rightarrow \mathbb{R}_{*}^{+}$. This naturally gives rise to a distance function $d_{\ell}$ between the points of $G$, and we let $|\mathrm{G}|_{\ell}$, also called $\ell-T O P$, denote the corresponding metric space.

To make this more precise, for each edge $e \in E(G)$ fix a homeomorphism $\sigma_{e}$ from $e$ to the real interval $[0, \ell(e)]$; by means of $\sigma_{e}$, any half-edge $f$, i.e. any connected subset of an edge, with endpoints $a, b$ obtains a length $\ell(f)$, namely $\ell(f):=\left|\sigma_{e}(a)-\sigma_{e}(b)\right|$. Now use $\ell$ to define a distance function on $G$ : for any $x, y \in V(G)$ let $d_{\ell}(x, y)=\inf _{P}$ is an $x-y$ path $\ell(P)$, where $\ell(P):=\sum_{e \in E(P)} \ell(e)$. For points $x, y \in G$ that might lie in the interior of an edge we define $d_{\ell}(x, y)$ similarly, but instead of graph-theoretical paths we consider arcs in the 1-complex $G$ : let $d_{\ell}(x, y)=\inf _{P}$ is an $x-y$ arc $\left(\sum_{f}\right.$ is an edge or half-edge in $\left.P(f)\right)$. By identifying any two vertices $x, x^{\prime}$ of $G$ for which $d_{\ell}\left(x, x^{\prime}\right)=0$ holds we obtain a metric space $\left(\bar{G}, d_{\ell}\right)$. Note that if $G$ is locally finite then $\bar{G}=G$. Let $|\mathrm{G}|_{\ell}$ be the completion of $\left(\bar{G}, d_{\ell}\right)$.

The boundary points of $G$ are the elements of the set $\partial^{\ell} G:=|\mathrm{G}|_{\ell} \backslash \pi(\bar{G})$, where $\pi$ is the canonical embedding of $\bar{G}$ in its completion $|\mathrm{G}|_{\ell}$.

The space $|G|_{\ell}$ was introduced in [16, where several important special cases 
were found, and many basic facts were proved. Here we list some of these facts that we are going to use in this paper. For the first of them, it is easy to realize the connection to the current paper by interpreting the resistance of an edge as its length:

finl Theorem 2.2 ([16]). If $\sum_{e \in E(G)} \ell(e)<\infty$ then $|G|_{\ell} \approx|G|$.

(Where " $\approx$ " means that the identity on $G$ extends to a homeomorphism between the two spaces.)

For our next lemma, fix an enumeration $e_{0}, e_{1}, \ldots$ of $E(G)$, and let $E_{n}:=$ $\left\{e_{0}, \ldots, e_{n}\right\}$. Moreover, let $\stackrel{\circ}{e}_{n}$ denote the set of inner points of the edge $e_{n}$, and let $\dot{E}_{n}:=\bigcup\left\{\stackrel{\circ}{0}_{0}, \ldots, \stackrel{\circ}{n}_{n}\right\}$.

epsNLF Lemma $2.3(16)$. Let $C$ be a circle or arc in $|G|_{\ell}$ such that $E(C)$ is dense in $C$. Then, for every $\epsilon \in \mathbb{R}^{+}$there is an $n \in \mathbb{N}$ such that for every subarc of $C$ in $|G|_{\ell} \backslash E_{n}$ connecting two vertices $v, w$ there holds $d_{\ell}(v, w)<\epsilon$.

lcisle Lemma 2.4 (16]). If $\sum_{e \in E(G)} \ell(e)<\infty$ then for every circle or arc $C$ in $|G|_{\ell}$ there holds $l(C)=\sum_{e \in E(C)} \ell(e)$.

\section{Intermediate results}

\subsection{Splitting infinite circles into cycles}

In this section we show that it is possible to write any infinite circle in a graph as a sum of a sparse family of finite cycles. We will later use this fact to show that the infinite circles of a network must also satisfy $(\overline{K 2})$ if all finite ones do, which will play an important role in the proof of our main result in Section 5 .

Call a family of edge sets sparse if no edge appears in more than three members of the family.

sparse Lemma 3.1. Let $G$ be a countable graph and let $C$ be a directed circle in $|G|$. Then there is a sparse family $\left\{D_{n}\right\}_{n \in \mathbb{N}}$ of finite directed cycles $D_{n}$ in $G$ such that $\vec{E}(C)=\sum_{n} \vec{E}\left(D_{n}\right)$,

where the latter sum is a formal sum of directed edges with coefficients in $\mathbb{Z}$ in which two edges cancel out if they correspond to the same edge with opposite orientations. (Lemma 3.1 was proved implicitely also in 11.)

Proof. Let $e_{0}, e_{1}, \ldots$ be an enumeration of $E(G)$ and let $\vec{f}_{0}, \vec{f}_{1}, \ldots$ be an enumeration of $\vec{E}(C)$. Pick an assignment $\ell: E(G) \rightarrow \mathbb{R}_{*}^{+}$such that $\sum_{e \in E(G)} \ell(e)<\infty$, and recall that by Theorem 2.2 there holds $|\mathrm{G}|_{\ell} \approx|G|$.

We will construct $\left\{D_{n}\right\}_{n \in \mathbb{N}}$ recursively, in $\omega$ steps. To begin with, Let $D_{0}$ be any finite cycle containing $f_{0}$, directed in the direction of $\vec{f}_{0}$. Combined with $C$ this cycle gives rise to what we call a quasi-circle: that is, a continuous image of $S^{1}$ comprising a finite path (in this case $D_{0} \backslash f_{0}$ ), called the green part of the quasi-circle, and a subarc of $C$ (in this case $C \backslash f_{0}$ ), called its blue part. Note that the green and blue part of a quasi-circle might have some common vertices and edges. We now construct the other $D_{i}$ inductively, in $\omega$ steps. For $i=1,2, \ldots$, suppose we have already constructed $D_{0}, \ldots D_{i-1}$, and specified a set $\mathcal{Q}_{i-1}$ of quasi-circles (let $\mathcal{Q}_{0}$ be the singleton containing the above quasi-circle) so that 
(i) for every $\vec{f} \in \vec{E}(C)$, either $\vec{f} \in \sum_{j<i} \vec{E}\left(D_{j}\right)$ (in the right direction) or $f$ is contained in the blue part of some quasi-circle in $\mathcal{Q}_{i-1}$;

sii (ii) for any two distinct elements $L, M$ of $\mathcal{Q}_{i-1}$, the blue parts of $M$ and $L$ are disjoint and the green parts of $M$ and $L$ are edge-disjoint.

siii (iii) the green part of every element of $\mathcal{Q}_{i-1}$ is contained in $\bigcup_{j<i} D_{j}$.

Then, in step $i$, let $k=k(i)$ be the least index such that $\vec{f}_{k} \notin \sum_{j<i} \vec{E}\left(D_{j}\right)$ (we choose this $k$ even if $\overleftarrow{f_{k}} \in \sum_{j<i} \vec{E}\left(D_{j}\right)$ ). By (i) there is an $M=M(i) \in \mathcal{Q}_{i-1}$ the blue part of which contains $f_{k}$, and by (ii) this $M$ is unique.

Let $h=h(i):=\min \left\{\ell(e) \mid e \in \bigcup_{i<i} E\left(D_{j}\right) \cup\left\{f_{k}\right\}\right\}$. By our choice of $\ell$ and Lemma 2.2, we can apply Lemma 2.3 to $C$, which yields an $n=n(i)$ such that for every subarc of $C$ in $\left.|\mathrm{G}|_{\ell}\right\rangle E_{n}$ connecting two vertices $v, w$ there holds $d_{\ell}(v, w)<h$. As the blue part $M_{b}$ of $M$ is, by construction, a subarc of $C$, the latter assertion also holds for $M_{b}$. Let $x, y$ be the endpoints of $M_{b}$, and let $M_{b}^{x}$ (respectively, $M_{b}^{y}$ ) be the component of $M_{b} \backslash f_{k}$ containing $x$ (resp. $y$ ). We may assume without loss of generality that

$$
h<d\left(M_{b}^{x}, M_{b}^{y}\right) / 2,
$$

where $d\left(M_{b}^{x}, M_{b}^{y}\right)$ is the minimum distance of a point in $M_{b}^{x}$ from a point in $M_{b}^{y}$, for otherwise we could have chosen a smaller bound than $h$ before applying Lemma 2.3.

Our aim now is to construct the directed trail $D_{i}$ that contains $\vec{f}_{k}$ as well as the green part $M_{g}$ of $M$, the latter traversed in the opposite direction. To achieve this, we need to construct two paths $P_{x}, P_{y}$, edge-disjoint from each other and from $M_{g}$, each path joining an endvertex of $f_{k}$ to an endvertex of $M_{g}$.

To construct $P_{x}$, let $\vec{g}_{1}, \ldots, \vec{g}_{r}$ be an enumeration of the directed edges in $E_{n} \cap E\left(M_{b}^{x}\right)$ in the order and orientation they appear on $M_{b}^{x}$ as we move from $f_{k}$ to $x$. Let $P_{x}^{1}$ be a path from the endvertex of $f_{k}$ in $M_{b}^{x}$ to the tail of $\vec{g}_{1}$, and for every $1<j \leq r$ let $P_{x}^{j}$ be a path from the head of $\vec{g}_{j}$ to the tail of $\vec{g}_{j+1}$. Finally, let $P_{x}^{r+1}$ be a path from the head of $\vec{g}_{r}$ to $x$ (some of these paths may be trivial). We can now combine these paths with the edges $g_{1}, \ldots, g_{r}$ to obtain a directed $f_{k}-x$ walk, and shortcutting this walk if necessary we can transform it into a path, which path we call $P_{x}$. Note that shortcutting a walk does not influence the order and direction in which the remaining edges are traversed. We construct $P_{y}$ similarly. Note that by our choice of $E_{n}$, we could have chosen the paths $P_{x}^{j}$ so that $\ell\left(P_{x}^{j}\right)<h$ for every $j$. This, and the choice of $h$, implies that no $P_{x}^{j}$ can contain an edge in $\bigcup_{j<i} D_{j}$ as any such edge is longer than $P_{x}^{j}$, from which we obtain that

$$
P_{x} \cup P_{y} \text { contains no edge in }\left(\bigcup_{j<i} D_{j}\right) \backslash\left(E_{n} \cap E(C)\right) .
$$

Moreover, by (2) we may assume that $P_{x}$ and $P_{y}$ are disjoint; indeed, if some $P_{x}^{j}$ has a vertex in common with some $P_{y}^{j^{\prime}}$, then their union $P_{x}^{j} \cup P_{y}^{j^{\prime}}$ contains an $M_{b}^{x}$ $M_{b}^{y}$ path of length at most $\ell\left(P_{x}^{j}\right)+\ell\left(P_{y}^{j^{\prime}}\right)<2 h<d\left(M_{b}^{x}, M_{b}^{y}\right)$, a contradiction.

Let $D_{i}:=\vec{f}_{k} P_{x}\left(-M_{g}\right)\left(-P_{y}\right)$, where $(-P)$ denotes the path $P$ traversed in the inverse direction. Note that $D_{i}$ is not necessarily a cycle, as we would like it 
to be, but rather a closed walk, but we will later modify it into a sum of cycles. By the construction of $D_{i}$ we have

$D_{i}$ traverses $M_{g}$, and does so in the opposite direction as $M$ does.

Moreover, $D_{i}$ traverses no edge in $E(G) \backslash E(C)$ more than once and traverses each edge in $E(C)$ at most twice (the latter can occur for an edge that happens to lie in $\left.E_{n} \cap E(C) \cap M_{g}\right)$.

To complete step $i$, it remains to define $\mathcal{Q}_{i}$. To obtain $\mathcal{Q}_{i}$ from $\mathcal{Q}_{i-1}$, we remove $M$ and add the quasi-circles obtained as follows. Consider the subspace $M_{b}^{x} \backslash\left(E\left(P_{x}\right) \cap\left\{\stackrel{\circ}{g}_{1}, \ldots, \stackrel{\circ}{g}_{r}\right\}\right)$ of $M_{b}^{x}$; note that for every component $K$ of this subspace there is a subpath $P_{K}$ of $P_{x}$ connecting the endvertices of $K$. Now $K \cup P_{K}$ defines a quasi-circle whose blue part is $K$ and whose green part is $P_{K}$. Similarly for $M_{b}^{x}$ and $P_{y}$. Add all these quasi-circles to $\mathcal{Q}_{i-1} \backslash M$ to obtain $\mathcal{Q}_{i}$. It follows from our choice of $P_{K}$, (iii) and (3) that

no edge appears in the green part of more than one element of $\mathcal{Q}:=$ $\cup \mathcal{Q}_{i}$.

Moreover, it is easy to check that

$D_{i}$ is the edge-disjoint union of edges in $\left\{g_{1}, \ldots, g_{r}\right\} \subset E(C)$ and green parts of elements of $\mathcal{Q}_{i}$.

Finally, it is easy to see that (i), (ii) and (iii) are all satisfied by $\mathcal{Q}_{i}$ if they were satisfied by $\mathcal{Q}_{i-1}$ (which is the case by our inductive hypothesis).

We have thus constructed the family $\left\{D_{n}\right\}_{n \in \mathbb{N}}$, and by (5) this family has the desired property that no edge is traversed more than three times (in fact, an edge in $E(G) \backslash E(C)$ is traversed either twice (in opposite directions) or not at all, and an edge in $E(C)$ is traversed either once or three times). Note that by our choice of the edge $f_{k}$, every edge of $E(C)$ will eventually appear in the sum $\sum_{j<i} \vec{E}\left(D_{j}\right)$, and in fact with the right orientation, for some step $i$. It also follows from our choice of the edge $f_{k}$ that every quasi-circle in $\mathcal{Q}$ will be considered as $M(i)$ for some step $i$, and so by (4) its green part will eventually disappear from the sum of the $D_{n}$. It follows from these observations and (6) that $\sum_{n \in \mathbb{N}} \vec{E}\left(D_{n}\right)=\vec{E}(C)$.

Thus, the family $\left\{D_{n}\right\}_{n \in \mathbb{N}}$ has all the desired properties except that $D_{n}$ is not necessarily a cycle but rather a closed walk that might traverse some edges twice and visit vertices more often. This, however, is easy to amend: if $D_{n}$ visits some vertex $x$ more than once, then we can split it into closed subwalks that each visit $x$ only once, while traversing each edge in the same direction as $D_{n}$ does. Performing this operation recursively, we can split $D_{n}$ into a family $D_{1}^{\prime}, \ldots D_{k}^{\prime}$ of directed cycles such that $\sum \vec{E}\left(D_{j}^{\prime}\right)=\vec{E}\left(D_{n}\right)$, and so replacing each $D_{n}$ by such a family we obtain the desired result.

Interestingly, in the last proof we used $\ell-T O P$ to prove an assertion that at first sight does not seem to be related to it. I would be interested to see a graph-theoretical proof of Lemma 3.1

Lemma 3.1 motivates the following problem.

Problem 3.1. Let $G$ be a locally finite graph, and let $C$ be a circle in $|G|$. Prove that $G$ has a planar subgraph $H$ containing $E(C)$ such that $E(C)$ is also the edge-set of a circle in $|H|$ (so that $H$ can be drawn in the plane with $E(C)$ bounding a face). 


\subsection{Shortcutting topological paths}

It is a well-known and useful fact that the image of a topological path in a Hausdorff space contains an arc with the same endpoints [20. For our uniqueness proof in Section 5 we will need a stronger version of this fact, saying, intuitively, that this arc can be chosen so that its points are traversed in the same order as in the original topological path. In order to state this more formally, let $\sigma, \tau:[0,1] \rightarrow X$ be topological paths in a Hausdorff space $X$. We say that $\tau$ shortcuts $\sigma$ if there is a monotone increasing injection $m:[0,1] \rightarrow[0,1]$ such that $\tau(x)=\sigma \circ m(x)$ for every $x \in[0,1]$. We will prove that

oriarc Lemma 3.2. Let $\sigma:[0,1] \rightarrow X$ be a topological path in a Hausdorff space $X$, and suppose its endpoints $u:=\sigma(0), v:=\sigma(1)$ are distinct. Then, there is an injective topological $u-v$ path in $X$ that shortcuts $\sigma$.

For this we are going to need the following

hall Lemma 3.3 (20, Lemmas 5.12, 5.13]). Let $X$ be a Hausdorff space, and let $\sigma:[0,1] \rightarrow X$ be a topological path in $X$. Then, there exists a closed subset $F$ of $[0,1]$ such that

hi (i) $\sigma(0), \sigma(1) \in \sigma(F)$;

hii (ii) If $C$ is any component of $[0,1] \backslash F$ and $x, y$ are the end points of $C$, then $\sigma(x)=\sigma(y)$

(iii) No proper closed subset of F satisfies both (i) and (ii), and

hiv (iv) $\sigma(F)$ is an arc.

We now proceed with the proof of Lemma 3.2

Proof. Apply Lemma 3.3 to $\sigma$ to obtain a subset $F$ of $[0,1]$ with properties (i) (iv) By (iv) $A:=\sigma(F)$ is an arc, so let $b: A \rightarrow[0,1]$ be a homeomorphism. Define the metric $d_{A}$ on $A$ by $d_{A}(x, y):=|b(x)-b(y)|$; clearly, $d_{A}$ is compatible with the topology of $A$.

We now define a mapping $\sigma^{\prime}:[0,1] \rightarrow X$ that will be an intermediate step towards the construction of $\tau$. For every point $x \in F$ let $\sigma^{\prime}(x)=\sigma(x)$. For every component $C$ of $[0,1] \backslash F$, recall that by (ii) $\sigma(x)=\sigma(y)=p$ where $x, y$ are the end points of $C$, and let $\sigma(z):=p$ for every $z \in C$. Obviously, $\sigma^{\prime}$ is a topological path. Moreover, it is easy to see that $\sigma^{\prime}$ shortcuts $\sigma$; indeed, just define $m:[0,1] \rightarrow[0,1]$ to map any point $x \in F$ to itself and any point $z \notin F$ to the endpoint of the component of $[0,1] \backslash F$ in which it lies.

Note that $\sigma^{\prime}$ is almost injective, that is, the preimage of any point is an interval of $[0,1]$. This implies that the length $h$ of $\sigma^{\prime}$ with respect to $d_{A}$ is finite, since, easily, any almost injective path in $[0,1]$ has finite length. Thus we may define a mapping $\tau:[0,1] \rightarrow \sigma(F)$ by mapping any point $x \in[0,1]$ to a point $\sigma^{\prime}(y)$ such that the restriction $\sigma^{\prime} \uparrow[0, y]$ of $\sigma^{\prime}$ to $[0, y]$ has length $x / h$ (with respect to $\left.d_{A}\right)$. Note that by the definition of the length of a topological path $\tau$ is well defined, i.e. if $y, y^{\prime} \in[0,1]$ are such that $l\left(\sigma^{\prime} \uparrow[0, y]\right)=l\left(\sigma^{\prime}\left\lceil\left[0, y^{\prime}\right]\right)\right.$ then $\sigma^{\prime}(y)=\sigma^{\prime}\left(y^{\prime}\right)$. It is straightforward to check that $\tau$ shortcuts $\sigma^{\prime}$, and thus $\tau$ also shortcuts $\sigma$ since $\sigma^{\prime}$ shortcuts $\sigma$. Note that $\tau(0)=u$ and $\tau(1)=v$. 
It is also easy to prove that $\tau$ is injective; for if $x \leq y \in[0,1]$ then $\sigma^{\prime}$ contains a topological path $\sigma^{\prime \prime}$ from $\tau(x)$ to $\tau(y)$ such that $l\left(\sigma^{\prime \prime}\right)=(y-x) / h>0$, and as $\sigma^{\prime \prime}$ is almost transitive this means that its endpoints $\tau(x), \tau(y)$ are distinct.

We claim next that $\tau$ is continuous. For this, pick a point $x \in[0,1]$ and let $O$ be an open ball of radius $\epsilon \in \mathbb{R}^{+}$with respect to $d_{A}$ around $\tau(x)$. We have to show that $[0,1]$ has an open set $U \ni x$ such that $\tau(U) \subseteq O$. But this is easy: let $U:=\left[x-\frac{\epsilon}{h}, x+\frac{\epsilon}{h}\right]$. For every $y \in U$ there is a topological $\tau(x)$ $\tau(y)$ path $\sigma^{\prime \prime}$ contained in $\sigma^{\prime}$ such that $l\left(\sigma^{\prime \prime}\right)=(y-x) / h$. As the length of any topological path is, by definition, at least the distance of its endvertices, we have $d_{A}(\tau(x), \tau(y)) \leq(y-x) / h$, and thus $\tau(y) \in O$. Since $y$ was arbitrary, we have $\tau(U) \subseteq O$ as required. This proves that $\tau$ is continuous.

\section{Existence}

The existence of a flow of finite energy satisfying Kirchhoff's cycle law in a locally finite network is a well-known fact, and there are several standard techniques to prove it. Here we will see two such techniques, and point out that they can also be employed when looking for a non-elusive flow with the above properties. Both these techniques start by showing that in any network $N=(G, r, p, q, I)$, where $G$ is locally finite, there is a $p-q$ flow $i$ of intensity $I$ that has minimum energy among all such flows; it is then an easy step to show that $i$ satisfies Kirchhoff's cycle law: if $C$ were a directed cycle with $\sum_{\vec{e} \in \vec{E}(C)} v(\vec{e})>0$, then subtracting from $i$ a constant circular flow around $C$ of sufficiently small intensity yields a flow $i^{\prime}$ with $W\left(i^{\prime}\right)<W(i)$, a contradiction (note that $W(i)<\infty$ since there are flows of finite energy in $N$ : just pick a $p-q$ path $P$ and send a constant flow of intensity $I$ along $P$ ).

Thus the interesting part is to show the existence of a (non-elusive) flow of minimum energy. Our first technique does so using the following well-known fact.

hilb Lemma 4.1 ([27, Theorem 4.10]). If $C$ is a non-empty, closed, convex subset of a Hilbert space, then there is a unique point $y \in C$ of minimum norm among all elements of $C$.

In order to apply it, let $H$ be the space of all functions $f: E(G) \rightarrow \mathbb{R}$ endowed with the inner product $\langle f, g\rangle:=\sum_{e \in E(G)} f(e) r(e) g(e)$, and note that the corresponding norm is $\|f\|=\sqrt{W(f)}$. It is straightforward to check that this is a Hilbert space, and that the subset $C$ consisting of non-elusive flows is closed and convex. Thus Lemma 4.1 yields a non-elusive flow of minimum energy.

Our second technique uses a standard compactness argument. Recall that there is at least one flow in $N$ with finite energy $W$. This $W$ yields, for every edge $e$, an upper bound for the amount of flow $i(e)$ that a flow $i$ of minimum energy, if one exists, can ever send along $e$ : this upper bound is $\hat{i}(e):=\sqrt{W / r(e)}$. Now consider for every edge $e$ a topological space $X_{e}$ homeomorphic to the real interval $[-\hat{i}(e), \hat{i}(e)]$, and let $X:=\Pi_{e} X_{e}$ be the product of these spaces. Every (non-elusive) flow $f$ in $N$ can be represented as a point $p_{f}$ in $X$ : from each component $X_{e}$ choose the point of $[-\hat{i}(e), \hat{i}(e)]$ corresponding to $f(e)$ (we are assuming a choice of an orientation for each edge). Let $\left(f_{i}\right)_{i \in \mathbb{N}}$ be a sequence of non-elusive flows in $N$ whose energies converge to the infimum $M$ of the energies 
of all such flows. By Tychonoff's theorem, $X$ is a compact space; thus, $\left(f_{i}\right)_{i \in \mathbb{N}}$ has an accumulation point $w$ in $X$, and it is straightforward to check that the function $i: E(G) \rightarrow \mathbb{R}$ corresponding to $w$ is a flow and in fact a non-elusive one. It is also not hard to see that $W(i)=M$.

We have thus given two proofs of the existence part of our main result:

Texist Theorem 4.2. Let $N=(G, r, p, q, I)$ be a locally finite network. Then there is a unique non-elusive flow $i$ of minimum energy in $N$. This flow satisfies Kirchhoff's cycle law.

(The uniqueness of $i$ only follows from our first proof.)

Next, we show that the flow $i$ provided by Theorem 4.2 can be obtained as a limit of electrical currents in a sequence of finite networks converging to $N$. The aim of this fact is a better understanding of the concept of non-elusive flows, but the reader may choose to skip to the next section as we will not make explicit use of this fact later.

Let $G_{n}, n \in \mathbb{N}$, be the (finite) subgraph of $G$ spanned by the vertices at distance at most $n$ from $p$, and let $G_{n}^{* *}$ be the graph obtained from $G$ by contracting each component $K$ of $G-G_{n}$ into a vertex $v_{K}$ (keeping multiple edges that may result from these contractions). We will, with a slight abuse, use $G_{n}$ and $G_{n}^{* *}$ to also denote the corresponding networks, not just the graphs. For a finite network $H$ we denote by $i(H)$ the unique electrical current in $H$.

Proposition 4.3. Let $N$ be locally finite network, and let $i$ be the non-elusive flow in $N$ with minimum energy. Then $i=\lim _{n} i\left(G_{n}^{* *}\right)$; in particular, the latter limit exists.

Proof. Define $i_{n}^{* *}: E\left(G_{n}^{* *}\right) \rightarrow \mathbb{R}$ by $i_{n}^{* *}(e)=i(e)$ for every $n$; since $i$ is nonelusive, it follows that $i_{n}^{* *}$ satisfies Kirchhoff's node law for every contracted vertex. Thus $i_{n}^{* *}$ is a $p-q$ flow of value $I$ in $G_{n}^{* *}$, and thus $W\left(i\left(G_{n}^{* *}\right)\right) \leq W\left(i_{n}^{* *}\right) \leq$ $W(i)$ for every $n$.

Let $g$ be an accumulation point of the sequence $\left(i\left(G_{n}^{* *}\right)\right)_{n \in \mathbb{N}}$ in the product space $X$ defined as above; here we have to check whether the components $X_{e}$ are large enough that $X$ can accommodate the elements of the latter sequence, but this is indeed the case since we have shown that $W\left(i\left(G_{n}^{* *}\right)\right) \leq W(i)$ for every $n$. The latter inequality also implies

$$
W(g) \leq W(i) .
$$

As in our compactness proof of Theorem4.2 it is easy to prove that $g$ is a flow in $N$ satisfying Kirchhoff's cycle law. We moreover claim that $g$ is non-elusive. Indeed, for every finite cut $\left(X, X^{\prime}\right)$ that does not separate $p$ from $q$ there is an $n \in \mathbb{N}$ such that every edge in $\left(X, X^{\prime}\right)$ is contained in $G_{m}^{* *}$ for every $m>n$. Since every flow in a finite network is non-elusive, $g$ is an accumulation point of flows $i_{n}$ satisfying $i_{n}\left(X, X^{\prime}\right)=0$; thus $g\left(X, X^{\prime}\right)=0$ holds as desired. By Theorem 4.2 and (77) we obtain $g=i$. In particular, $g=i$ is the only accumulation point of the sequence $\left(i\left(G_{n}^{* *}\right)\right)_{n \in \mathbb{N}}$, and we may write $i=\lim _{n} i\left(G_{n}^{* *}\right)$.

The last result motivates the following problem.

Conjecture 4.1. If $\lim i\left(G_{n}\right)=\lim i\left(G_{n}^{* *}\right)$ then there is a unique flow in $N$ satisfying Kirchhoff's cycle law. 


\section{The main result: Uniqueness}

suniq As mentioned in the introduction, in order to prove the uniqueness of a nonelusive flow in $N$ satisfying Kirchhoff's cycle law, we will consider the difference $z$ of two distinct hypothetical flows of this kind; we will not be able to find a cycle in $z$ as in the finite case, but instead we will be able to find a circle. In order to obtain a contradiction, we then need to show that circles must satisfy (K2) as well. This is an immediate corollary of Lemma 3.1 and the following easy property of real vectors.

vectors Lemma 5.1. Let $R, I$ be two infinite-dimensional vectors with positive real values. If $\langle I, R\rangle>\langle\mathbb{1}, R\rangle$ then $\left\langle I^{2}, R\right\rangle>\langle I, R\rangle$.

(where $\langle$,$\rangle denotes the usual inner product, \mathbb{1}$ denotes the all-ones vector and $I^{2}$ denotes the vector whose value at each coordinate is the square of the value of $I$ at that coordinate).

Proof. For every component $j$, if there is a gain in this component when going from $\langle\mathbb{1}, R\rangle$ to $\langle I, R\rangle$, that is, if $I(j) R(j) \geq R(j)$, then this gain becomes even bigger when going from $\langle I, R\rangle$ to $\left\langle I^{2}, R\right\rangle$. On the other hand, if there is a loss in this component when going from $\langle\mathbb{1}, R\rangle$ to $\langle I, R\rangle$, then the loss becomes smaller when going from $\langle I, R\rangle$ to $\left\langle I^{2}, R\right\rangle$, since $I(j) R(j)<R(j)$ in that case. The assertion follows easily from these two observations.

tinfkir Corollary 5.2. Let $N$ be a locally finite electrical network with resistances $r$ : $E(G) \rightarrow \mathbb{R}_{*}^{+}$such that $\sum_{e \in E(G)} r(e)<\infty$. Let $i$ be a flow satisfying Kirchhoff's cycle law and $W(i)<\infty$. Then for every directed circle $C$ in $|G|$ there holds $\sum_{\vec{e} \in \vec{E}(C)} v(\vec{e})=0$; in particular, the latter sum is well-defined.

Proof. Lemma 3.1 yields a sparse family $\left\{D_{n}\right\}_{n \in \mathbb{N}}$ of finite directed cycles $D_{n}$ in $G$ such that $\vec{E}(C)=\sum_{n} \vec{E}\left(D_{n}\right)$. By $\underline{K 2}$ we have $\sum_{\vec{e} \in \vec{E}\left(D_{n}\right)} v(\vec{e})=0$ for every $n$. Thus it suffices to prove that the sum $\sum_{n} \sum_{\vec{e} \in \vec{E}\left(D_{n}\right)} v(\vec{e})$ is absolutely convergent. To prove this, recall that we are assuming that $\sum_{e \in E(G)} r(e)<\infty$ and $\sum_{e \in E(G)} i^{2}(e) r(e)<\infty$. It follows with Lemma 5.1 that $\sum_{e \in E(G)}|i(e)| r(e)=$ : $u<\infty$. Since the family $D_{n}$ is sparse, this means that $\sum_{n} \sum \vec{e} \in \vec{E}\left(D_{n}\right)|v(\vec{e})| \leq$ $3 u<\infty$, which proves that the sum is absolutely convergent as desired. Thus $\sum_{\vec{e} \in \vec{E}(C)} v(\vec{e})=\sum_{n} 0=0$.

The following lemma states that any infinite sequence of topological paths between two fixed vertices $x, y$ in $|G|$ has a "converging" subsequence whose limit contains a topological $x$ - $y$ path. More precisely, given a sequence $E_{1}, E_{2}, \ldots$ of sets, let us write

$$
\liminf \left(E_{n}\right):=\bigcup_{i \in \mathbb{N}} \bigcap_{j>i} E_{j}
$$

for the set of elements eventually in $E_{n}$.

hp Lemma $5.3([18])$. Let $G$ be a locally finite graph, let $x, y \in V(G)$ and let $\left(\tau_{n}\right)_{n \in \mathbb{N}}$ be a sequence of topological $x-y$ paths in $|G|$. Then, there is an infinite subsequence $\left(\tau_{a_{n}}\right)_{n \in \mathbb{N}}$ of $\left(\tau_{n}\right)$ and a topological $x-y$ path $\sigma$ in $|G|$ such that $E(\sigma) \subseteq \lim \inf \left(E\left(\tau_{a_{n}}\right)\right)$. Moreover, if no $\tau_{n}$ traverses any edge more than once then $\bar{E}(G) \backslash E(\sigma) \subseteq \liminf \left(E(G) \backslash E\left(\tau_{a_{n}}\right)\right)$, no edge is traversed by $\sigma$ more than 
once, and for every finite subset $F$ of $E(\sigma)$ there is an $m \in \mathbb{N}$ such that the linear ordering of $F$ induced by $\sigma$ coincides with that induced by $\tau_{a_{n}}$ for every $a_{n}>m$.

We can now finish the proof of our main result.

Proof of Theorem 1.1. By Theorem 4.2 there exists at least one such flow, so it only remains to prove the uniqueness of $i$. Suppose, to the contrary, there are two non-elusive flows $i \neq f$ of finite energy in $N$ that both satisfy Kirchhoff's cycle law, and put $z:=i-f$.

Since $i \neq f$, there is an oriented edge $\overrightarrow{e_{0}}$ with $z\left(\overrightarrow{e_{0}}\right)>0$. Let $G_{1} \subseteq G_{2} \subseteq$ $G_{3} \ldots$ be a sequence of finite subgraphs of $G$ such that $\bigcup_{n} G_{n}=G$ and $e_{0} \in$ $E\left(G_{1}\right)$.

Since both $i, f$ are non-elusive, so is $z$. Thus $z$ induces a circulation $z_{n}$ on $G_{n}^{* *}$ for every $n\left(G_{n}^{* *}\right.$ is defined as in Section 4 4 . Since $z_{n}$ a circulation in a finite network, there must exist an oriented cycle $C_{n}$ in $G_{n}^{* *}$ such that $\overrightarrow{e_{0}} \in \vec{E}\left(C_{n}\right)$ and $z(\vec{e})>0$ for every $\vec{e} \in E\left(C_{n}\right)$. As $z_{n}$ is induced from the circulation $z$ of $G$ that, clearly, satisfies Kirchhoff's cycle law, $C_{n}$ cannot be a cycle of $G$; thus $C_{n}$ contains a contracted vertex of $G_{n}^{* *}$. We are now going to make use of the sequence $\left(C_{i}\right)_{i \in \mathbb{N}}$ in order to construct an oriented circle $C$ in $|G|$ with $z(\vec{e})>0$ for every $\vec{e} \in E(C)$. For this, let $P_{n}$ be the (oriented) path $C-e_{0}$.

Applying Lemma 5.3 to $G$, letting $\tau_{n}$ be a topological path that traverses $P_{n}$ in a straight manner, we obtain a topological path $\sigma$, and we can concatenate $\sigma$ with $e_{0}$ to obtain a closed topological path $\sigma^{\prime}$, which we may assume traverses $e_{0}$ in the same direction as the $C_{n}$ do. By the second sentence of Lemma 5.3 . and since no $\tau_{n}$ visits a vertex more than once, $\sigma$ also visits no vertex more than once. Moreover, we may assume that every edge $e=x y \in E\left(\sigma^{\prime}\right)$ is traversed by $\sigma$ in the direction in which $z$ flows, in other words, $z(x, y)>0$ if $\sigma$ visits $x$ before $y$. Indeed, recall that each $P_{n}$ traverses its edges in the direction in which $z$ flows. To make sure that $\sigma$ traverses its edges in the same direction as the $P_{n}$ do, we can, before applying Lemma 5.3 to obtain $\sigma$, subdivide each edge $e=x y$ of $G$ by a dummy vertex $z$ into two edges $x z, z y$. Now the second sentence of Lemma 5.3 implies that if $x z, z y$ are traversed by $\sigma$ then they are traversed in the same order as in infinitely many of the $P_{n}$, which means that $\sigma$ traverses $e$ in the same direction as these $P_{n}$, which is the direction in which $z$ flows along $e$.

By Lemma 3.2, there is a circle $C$ that shortcuts $\sigma^{\prime}$. This circle clearly violates Corollary [5.2. since for one of its orientations $\vec{C}$ there holds $z(\vec{e})>0$ for every $\vec{e} \in E(\vec{C})$. This contradiction completes the proof.

Theorem 1.1 is best possible in the sense that we can not drop any of its requirements. Indeed, to see why the condition of being non-elusive is necessary, recall that the network of Figure 1 has several flows satisfying Kirchhoff's cycle law no matter how we choose its resistances.

To see that the condition $W(i)<\infty$ is necessary, consider the network $N$ of Figure 3. We will construct a non-trivial non-elusive $p-q$ flow $f$ of intensity 0 in $N$, i.e. a circulation, that satisfies Kirchhoff's cycle law. Let $f(p, q)=1$, and let $f$ also send a flow of value 1 along the two edges incident with $p, q$, so that $K 1$ is satisfied at both $p, q$. We can now assign a flow $f(e)$ to the perpendicular 
edge $e$ forming a 4-cycle $C$ with those three edges so that $C$ complies with K2). Then, we can assign a flow to each of the two edges incident with $e$ so that the endvertices of $e$ comply with (K1). Continuing like this, we obtain a function $f$ that satisfies both Kirchhoff laws and is non-elusive.

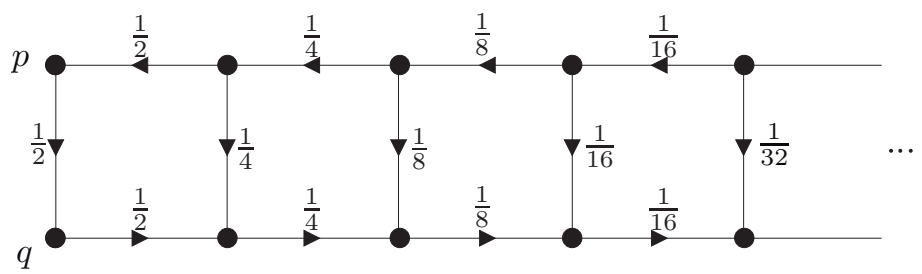

Figure 3: A network that has a non-constant non-elusive circulation satisfying Kirchhoff's cycle law. The numbers on the edges denote their resistances.

In view of Theorem 2.2 it is tempting to conjecture that the requirement $\sum r(e)<\infty$ in Theorem 1.1 can be replaced by the weaker requirement $|G|_{r} \approx$ $|G|$. However, in 11 we show a counterexample to this conjecture.

\section{Finite total conductance}

It is known that networks of finite total conductance have unique electrical currents. One way to prove this is to notice that in this case the corresponding random walk is recurrent 33. Lemma 4.2], which combined with Lyons' theorem (see [25] or 24]), and a little additional effort, implies uniqueness of electrical currents [28]. See Section 8 or [24, 33] for more on the relationship between electrical networks and random walks. In this section we give a non-probabilistic proof of this fact for the sake of completeness.

finc Theorem 6.1. Let $N=(G, r, p, q, I)$ be countable network with $\sum_{e \in E(G)} 1 / r(e)<$ $\infty$. Then there is a unique flow of finite energy satisfying Kirchhoff's cycle law. This flow is non-elusive.

Proof. Suppose there are distinct flows $f, g$ in $N$ both having finite energy and satisfying Kirchhoff's cycle law and consider their difference $z:=f-g$. It is not hard to check that the circulation $z$ has finite energy too, and it clearly also satisfies Kirchhoff's cycle law.

Pick an edge $f$ such that $z(f)>0$. Since $\sum_{e \in E(G)} 1 / r(e)<\infty$ and $\sum_{e \in E(G)} z^{2}(e) r(e)<\infty$, there is a finite set of edges $F \subset E(G)$ such that

$$
\sum_{e \in E(G) \backslash F}|z(e)|<|z(f)| .
$$

Let $U$ be the set of vertices incident with an edge in $F$, and let $G^{*}$ be the graph obtained from $G$ after identifying all vertices in $V(G) \backslash U$ into a single vertex $v^{*}$, keeping parallel edges if any arise. Note that $z$, considered as a function from $E(G)=E\left(G^{*}\right)$ to $\mathbb{R}$, is also a circulation in $G^{*}$; the $\operatorname{sum} \sum\{z(e) \mid$ e is incident with $\left.v^{*}\right\}$ is well-defined even if $v^{*}$ has infinite degree by (8), and it equals zero since the set $U$ is finite and every vertex in it satisfies $K 1$. We may assume that the graph $G^{*}$ has only finitely many edges, for if $x, y \in V\left(G^{*}\right)$ 
are joined by an infinite set of edges $B$, then we may replace all these edges by a single edge carrying the flow $\sum_{e \in B} z(e)$.

We claim that $z$ must traverse a cycle in $G^{*}$ that does not visit $v^{*}$. Indeed, since $z$ is a circulation in the finite graph $G^{*}$, it must traverse some cycle $C_{0}$. If $C_{0}$ visits $v^{*}$, then we can subtract from $z$ a constant circulation along $C_{0}$ to obtain a new circulation $z_{1}$ that does not traverse $C_{0}$. By (8) we have $z_{1}(f)>0$ since no edges incident with $v^{*}$ lie in $E(G) \backslash F$. If $z_{1}$ still traverses a cycle $C_{1}$ visiting $v^{*}$ we can subtract it to obtain $z_{2}$, and so on. Continuing like this we can, after finitely many steps, reach a circulation $z_{k}$ that traverses no cycle incident with $v^{*}$ and satisfies $z_{k}(f)>0$. But then, $z_{k}$ must traverse a cycle $C$ not incident with $v^{*}$, and by the construction of $z_{k}$ there follows that $z$ also traverses $C$. But $C$ is also a cycle in the original graph $G$, which contradicts the fact that $z$ satisfies Kirchhoff's cycle law.

Thus we have proved the uniqueness of a flow $i$ of finite energy satisfying Kirchhoff's cycle law. To prove that $i$ is non-elusive, it would now suffice to show the existence of a non-elusive flow $f$ in $N$ having finite energy and satisfying Kirchhoff's cycle law since uniqueness implies $f=i$. We already proved the existence of such a flow $f$ in Theorem 4.2 in the case that $G$ is locally finite. The interested reader will be able to check that our second proof of Theorem 4.2 also works in the non-locally-finite case if $\sum_{e \in E(G)} 1 / r(e)<\infty$-although not necessarily otherwise, see Section 7

\section{Non-locally-finite networks}

In this section we discuss how the results of this paper behave with respect to non-locally-finite graphs.

Let us start with an example. The graph $G$ of Figure 4 consists of two edges $e=p u, f=v q$ and an infinite family $\left\{P_{n}\right\}_{n \in \mathbb{N}}$ of independent $u-v$ paths of length two. Let us suppose that all edges in the $P_{n}$ have the same resistance, although this makes little difference. It is straightforward to check that the corresponding network does not have a flow of minimum energy (with a fixed intensity $I$ ), and in fact no flow that satisfies Kirchhoff's cycle law.

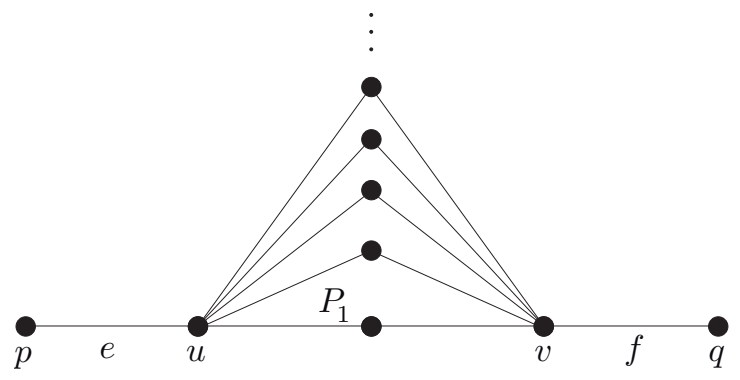

Figure 4: A simple example showing that non-locally-finite networks do not necessarily have flows satisfying Kirchhoff's cycle law.

This seems to suggest that the results of this paper cannot be extended to non-locally-finite graphs, but nevertheless there is a way around this: call a function $f: V^{2} \rightarrow \mathbb{R}$ a relaxed flow from $p$ to $q$ if it satisfies $f(x, y)=-f(y, x)$ for every $x, y, f(x, y)=0$ if $x y \notin E(G)$, and moreover it is non-elusive (defined 
as in Section 21). Note that if $G$ is locally finite then every relaxed flow is a nonelusive flow, since applying the non-elusiveness condition to the set of edges incident with a given vertex $x$ yields Kirchhoff's node law for $x$. It is worth mentioning that this definition effectively imposes Kirchhoff's node law to the points of $|G|$ rather than the vertices of $G$.

The interested reader will be able to show that Theorem 1.1 extends to nonlocally-finite graphs to assert the existence and uniqueness of a relaxed flow of finite energy if the total resistance is finite (and thus the graph countable). Indeed, all intermediate results we used are proved (either here or in the respective sources) for countable graphs as well, and the rest of the proof of Theorem 2.2 easily extends to countable graphs.

\section{Relationship to stochastic processes and the discrete Dirichlet Problem}

There is a well studied and fruitful relationship between electrical networks and random walks. Every electrical network gives rise to a random walk, in which the transition probabilities are proportional to the conductances of the corresponding edges and, conversely, every reversible irreducible Markov chain can be represented by an electrical network [24, 33.

It turns out that in a finite network the probability that random walk starting at a vertex $x$ reaches a fixed vertex $p$ before having visited another fixed vertex $q$ equals the potential of vertex $x$ induced by a voltage source imposing potential 1 at $p$ and potential 0 at $q$. It is natural to ask if this relationship carries over to the networks of the current paper, in other words, if the unique flow provided by Theorem 1.1 coincides with the flow induced by the corresponding random walk on the same graph. This is however not the case: in the graph of Figure 1 if the sum of the resistances is finite then the probability that random walk starting at a vertex $x \neq p$ to the left of $p$ visits $p$ before having visited $q$ is less than 1 , since such a random walk is transient and so the probability that $p$ is never visited is positive. It follows that the corresponding flow fails to be non-elusive.

It would be interesting though to try to define a continuous random process, i.e. a brownian motion, on $|G|_{r}$ in which the particle continues moving after reaching a boundary point of $|G|_{r}$; for such a process it might be the case that the corresponding flow is indeed non-elusive. To support this idea, we now show that given a network $N$ and a flow $f$ of finite energy in $N$ satisfying Kirchhoff's cycle law, it is possible to extend the potential function $P: V(G) \rightarrow \mathbb{R}$ from the vertices of $G$ to all of $|G|_{r}$ in a natural way. Recall that given a flow $i$ satisfying Kirchhoff's cycle law, one usually defines $P$ by fixing $P(q)=0$ and then letting $P(u)=\sum_{\vec{e} \in \vec{E}(R)} v(\vec{e})$, where $R$ is any path from $q$ to $u$; Kirchhoff's cycle law implies that $P(u)$ does not depend on the choice of $R$ but only on its endpoints. The following lemma allows us to extend $P$ to the boundary $\partial^{r} G$ of $|G|_{r}$ whenever we have a flow of finite energy satisfying Kirchhoff's cycle law. Note that for every such boundary point $x$ there is a ray of finite total resistance converging to $x$ in $|G|_{r}$, see [16, Lemma 4.1].

indep Lemma 8.1. Let $f$ be a flow of finite energy satisfying Kirchhoff's cycle law in a locally finite network $N=(G, r, p, q, I)$, let $x \in \partial^{r} G$, and let $R$ be a ray of 
finite total resistance starting at $q$ and converging to $x$. Then $\sum_{\vec{e} \in \vec{E}(R)} f(\vec{e}) r(\vec{e})$ is well defined and does not depend on $R$.

Proof (sketch). Well-definedness follows easily by Lemma 5.1. To show independence from $R$, let $R, S$ be two such rays. Since, easily, $R$ and $S$ belong to the same end of $G$, it is possible to find an infinite sequence $\left(P_{i}\right)_{i \in \mathbb{N}}$ of disjoint $R, S$ paths such that $\sum_{e \in \cup_{i} P_{i}} r(e)<\infty$, the endpoint of $P_{i}$ in $R$ comes after the endpoint of $P_{i-1}$ for every $i$, and similarly for the endpoints in $S$. The sum of potential differences $\sum_{e \in \cup_{i} P_{i}} f(e) r(e)$ is absolutely convergent by Lemma 5.1. Adding potential differences along all finite cycles formed by $R, S$ and a pair of subsequent $P_{i}$, and using the fact that these cycles satisfy $(\overline{K 2})$, we obtain $\sum_{\vec{e} \in \vec{E}(R)} f(\vec{e}) r(\vec{e})-\sum_{\vec{e} \in \vec{E}(S)} f(\vec{e}) r(\vec{e})=0$.

Thus we can extend $P$ to the boundary $\partial^{r} G$ by letting $P(x)=\sum_{\vec{e} \in \vec{E}(R)} f(\vec{e}) r(\vec{e})$ for some such ray $R$. The interested reader will be able to check that the function $P:|G|_{r} \rightarrow \mathbb{R}$ defined this way is continuous provided $f$ has finite energy.

Conversely, one may ask whether given any continuous potential function $P: \partial^{r} G \rightarrow \mathbb{R}$ on the boundary of $|G|_{r}$ there is a flow in $G$ inducing $P$. This is an instance of the well-known discrete Dirichlet Problem. Let us state it more precisely:

condir Conjecture 8.1. Let $G$ be a locally finite graph and fix a function $r: E(G) \rightarrow$ $\mathbb{R}^{+}$such that $|G|_{r}$ is compact. Then, for every continuous function $P: \partial^{r} G \rightarrow \mathbb{R}$ there is a circulation in $G$ that satisfies Kirchhoff's cycle law and induces $P$ in the above sense.

A typical instance of the discrete Dirichlet Problem presupposes a locally finite graph $G$ with a fixed compactification, usually the Freudenthal compactification $|G|$ or the hyperbolic compactification, and asks whether every continuous function on the boundary can be induced by a circulation in $G$; see for example 3, 21, 32. In most cases, all edges of $G$ are considered with resistance 1. In comparison, Conjecture 8.1 allows more flexibility as far as the choice of resistances and the choice of a compactification is concerned, but demands that these two choices are compatible with each other.

An interesting aspect of Conjecture 8.1 is the fact that every compact metric space is isometric to $\partial^{r} G$ for some appropriate choice of $G$ and $r$, see 16, Section 4].

Conjecture 8.1 has been proved by Carlson 9 for the special case that is perhaps most interesting from the point of view of the current paper: the case when $\sum_{e \in E} r(e)<\infty$.

\section{Acknowledgements}

I am very grateful to Wolfgang Woess for several discussions on the topic, in particular for drawing my attention to the applicability of Lemma 4.1 in this context, as well as for acquainting me with the proof sketch at the beginning of Section 6. I am also very grateful to Reinhard Diestel for motivating me to study electrical networks and for valuable discussions. 


\section{References}

mfmc [1] R. Aharoni, E. Berger, A. Georgakopoulos, A. Perlstein, and P. Sprüssel. The max-flow min-cut theorem for countable networks. To appear in J. Combin. Theory (Series B).

armstrong [2] M.A. Armstrong. Basic Topology. Springer-Verlag, 1983.

BeSchrHar [3] I. Benjamini and O. Schramm. Harmonic functions on planar and almost planar graphs and manifolds, via circle packings. Invent. math., 126:565$587,1996$.

biggs [4] N. L. Biggs. Algebraic potential theory on graphs. Bull. London Math. Soc., 29:641-682, 1997.

locFinTutte [5] H. Bruhn. The cycle space of a 3-connected locally finite graph is generated by its finite and infinite peripheral circuits. J. Combin. Theory (Series B), 92:235-256, 2004.

partition [6] H. Bruhn, R. Diestel, and M. Stein. Cycle-cocycle partitions and faithful cycle covers for locally finite graphs. J. Graph Theory, 50:150-161, 2005.

LocFinMacLane [7] H. Bruhn and M. Stein. MacLane's planarity criterion for locally finite graphs. J. Combin. Theory (Series B), 96:225-239, 2006.

degree [8] H. Bruhn and M. Stein. On end degrees and infinite circuits in locally finite graphs. Combinatorica, 27:269-291, 2007.

CarBou [9] R. Carlson. Boundary value problems for infinite metric graphs. In Analysis on graphs and its applications (Exner, Pavel et al, editors), volume 77 of Proceedings of Symposia in Pure Mathematics, pages 355-368. AMS, 2008.

diestelBook05 [10] R. Diestel. Graph Theory (3rd edition). Springer-Verlag, 2005.

Electronic edition available at:

http://www.math. uni-hamburg.de/home/diestel/books/graph.theory.

kirch2 [11] R. Diestel and A. Georgakopoulos. In preparation.

ends [12] R. Diestel and D. Kühn. Graph-theoretical versus topological ends of graphs. J. Combin. Theory (Series B), 87:197-206, 2003.

cyclesI [13] R. Diestel and D. Kühn. On infinite cycles I. Combinatorica, 24:68-89, 2004.

cyclesII [14] R. Diestel and D. Kühn. On infinite cycles II. Combinatorica, 24:91-116, 2004.

Freudenthal31

[15] H. Freudenthal. Über die Enden topologischer Räume und Gruppen. Math. Zeitschr., 33:692-713, 1931.

1top [16] A. Georgakopoulos. Graph topologies induced by edge lengths. arXiv.org 0903.1744, submitted.

fleisch [17] A. Georgakopoulos. Infinite hamilton cycles in squares of locally finite graphs. Adv. Math., 220:670-705, 2009. 
hp [18] A. Georgakopoulos. Topological circles and Euler tours in locally finite graphs. Electronic J. Comb., 16:\#R40, 2009.

geo [19] A. Georgakopoulos and P. Sprüssel. Geodetic topological cycles in locally finite graphs. Electronic J. Comb., 16:\#R144, 2009.

ElemTop [20] D.W. Hall and G.L. Spencer. Elementary Topology. John Wiley, New York 1955.

KaWoDir [21] V. Kaimanovich and W. Woess. The Dirichlet problem at infinity for random walks on graphs with a strong isoperimetric inequality. Probab. Theory Relat. Fields, 91:445-466, 1992.

KanaiRough2 [22] M. Kanai. Rough isometries, and combinatorial approximations of geometries of non- compact Riemannian manifolds. J. Math. Soc. Japan, 37:391413, 1985.

KanaiRough [23] M. Kanai. Rough isometries and the parabolicity of Riemannian manifolds. J. Math. Soc. Japan, 38:227-238, 1986.

LyonsBook [24] R. Lyons and Y. Peres. Probability on Trees and Networks. Cambridge University Press. In preparation, current version available at

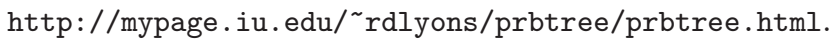

LyoSim [25] T. Lyons. A simple criterion for transience of a reversible Markov chain. Ann. Probab., 11:393-402, 1983.

CThyperb [26] S. Markvorsen, S. McGuinness, and C. Thomassen. Transient random walks on graphs and metric spaces with applications to hyperbolic surfaces. Proc. London Math. Soc., 64:1-20, 1992.

rudin [27] W. Rudin. Real and Complex Analysis. McGraw-Hill, 1974.

SchleInf [28] E. Schlesinger. Infinite networks and Markov chains. Boll. Unione Mat. Ital., VII. Ser., B, 6(1):23-37, 1992.

woessCurrents

[29] P. M. Soardi and W. Woess. Uniqueness of currents in infinite resistive networks. Discrete Appl. Math., 31(1):37-49, 1991.

hcs [30] M. Stein. Forcing highly connected subgraphs in locally finite graphs. J. Graph Theory, 54:331-349, 2007.

thomInfNet [31] C. Thomassen. Resistances and currents in infinite electrical networks. J. Combin. Theory (Series B), 49:87-102, 1990.

WoeDir [32] W. Woess. Dirichlet problem at infinity for harmonic functions on graphs. In International Conference on Potential Theory 1994, Proceedings (editors: J. Kral et. al.), pages 189-217. de Gruyter, Berlin, 1996.

WoessBook09 [33] Wolfgang Woess. Denumerable Markov chains. Generating functions, boundary theory, random walks on trees. EMS Textbooks in Mathematics. European Mathematical Society (EMS), Zürich, 2009. 